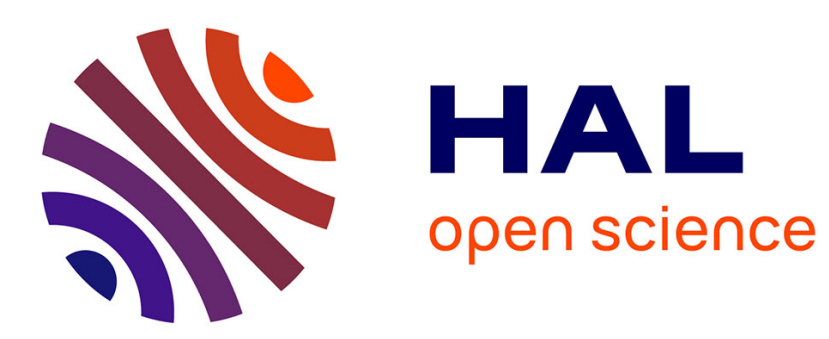

\title{
Effets associés et utilisation de gènes majeurs réduisant la taille chez la poule domestique \\ P. Merat
}

\section{To cite this version:}

P. Merat. Effets associés et utilisation de gènes majeurs réduisant la taille chez la poule domestique. Productions Animales, 1990, 3 (2), pp.151-158. hal-00895897

\section{HAL Id: hal-00895897 https://hal.science/hal-00895897}

Submitted on 1 Jan 1990

HAL is a multi-disciplinary open access archive for the deposit and dissemination of scientific research documents, whether they are published or not. The documents may come from teaching and research institutions in France or abroad, or from public or private research centers.
L'archive ouverte pluridisciplinaire HAL, est destinée au dépôt et à la diffusion de documents scientifiques de niveau recherche, publiés ou non, émanant des établissements d'enseignement et de recherche français ou étrangers, des laboratoires publics ou privés. 
INRA Prod. Anim.

1990, $3(2), 151-158$

\section{P. MERAT}

INRA Laboratoire de Génétique Factorielle 78350 Jouy-en-Josas

\section{Effets associés et utilisation de gènes majeurs réduisant la taille chez la poule domestique}

Résumé

Des gènes marqueurs identifiés par des critères qualitatifs (visuels, biochimiques ou sérologiques) peuvent montrer une association avec des caractères quantitatifs soit à cause d'un effet pléiotropique - situation où un gène influence plusieurs caractères - soit par suite d'un linkage avec d'autres gènes. Il est très difficile de faire la discrimination entre un effet pléiotropique et un linkage étroit, sauf lorsqu'une interprétation raisonnable de l'effet quantitatif attribué au gène marqueur peut être déduite de son effet primaire.

Des éléments permettant de distinguer un effet pléiotropique ou un linkage étroit d'une

Parmi les gènes majeurs réduisant la taille chez la poule, le gène lié au sexe $d w$ a fait l'objet de nombreuses études et d'applications en élevage. Son caractère récessif et le fait qu'il abaisse d'un tiers le poids de l'oiseau et de 20 à $25 \%$ sa consommation alimentaire l'ont fait utiliser pour " nanifier " des reproductrices " chair", qui donnent des poulets de taille normale en croisement avec une souche mâle standard. Pour la production d'oeufs, l'application au stade industriel présente plus de difficultés, du fait que dans les souches « ponte», surtout celles de petite taille (Leghorn), l'introduction de ce gène entraine une réduction du nombre d'oeufs et de leur poids moyen. En dépit de cela, dans les souches milourdes de type " oeuf brun », le gène $d w$ améliore l'efficacité alimentaire, évite le rationnement des pondeuses et réduit le pourcentage d'oeufs cassés ou sans coquille. Des travaux ont donc cherché à mieux cerner les conditions optimales d'utilisation de pondeuses "naines » : incorporer le gène de préférence dans des populations mi-lourdes, éviter la sous-consommation alimentaire, se placer dans des conditions de production où une économie sur l'aliment est particulièrement importante.

Un autre facteur lié au sexe issu de races Bantam, abaissant plus modérément la taille $(10-15 \%)$, sans effet réducteur du nombre d'oeufs, pourrait avoir une utilité dans des croisements particuliers. part, un linkage lâche d'autre part, sont le degré de persistance des effets associés au cours des générations pour une même population et leur degré de similitude entre populations différentes. Un effet pléiotropique est constamment attaché à un gène, alors qu'un effet par l'intermédiaire d'un linkage pourra être inversé dans certains groupes génétiques. Cependant, des interactions entre le gène en question et le reste du génome ou l'environnement peuvent aussi entrer en ligne de compte, comme l'indique le tableau 1.

Une autre approche pour rechercher des gènes individualisés ayant un effet important sur des caractères quantitatifs consiste à les déceler à partir de l'examen de paramètres statistiques, notamment existence de plusieurs modes ou de déviations des distributions de fréquences vis-à-vis de la normalité, hétérogénéité des variances selon les génotypes parentaux pour le ou les gènes majeurs supposés. Diverses méthodes ont été proposées. Ces méthodes ont un faible pouvoir de discrimination, et même si l'existence de gènes à effet propre important est suggérée, le problème d'identifier les génotypes individuels n'est souvent pas résolu. Chez les oiseaux domestiques l'application de telles méthodes n'a pas conduit jusqu'ici à des résultats significatifs.

Dans une brève revue des effets quantitatifs associés à des gènes marqueurs chez les volailles (limitée à l'espèce Gallus Gallus), nous choisirons ceux qui paraissent le plus susceptibles d'applications, ou les plus intéressants sur le plan scientifique. Cet article concerne des gènes réducteurs de la taille. 
Tableau 1. Discrimination entre pléiotropie et linkage.

\begin{tabular}{|c|c|}
\hline Effets & Hypothèses \\
\hline Constants au cours des générations successives & Pléiotropie ou linkage étroit \\
\hline $\begin{array}{l}\text { Variables (décroissants au cours } \\
\text { des générations) }\end{array}$ & \\
\hline - Population génétiquement stable & Linkage \\
\hline $\begin{array}{l}\text { - Population changeant génétiquement } \\
\text { avec le temps }\end{array}$ & $\begin{array}{l}\text { Linkage ou pléiotropie avec interaction } \\
\text { gène } \times \text { génome }\end{array}$ \\
\hline Constants dans des populations diverses & Pléiotropie \\
\hline Variables entre populations & $\begin{array}{l}\text { Linkage ou pléiotropie avec interaction } \\
\text { gène } \times \text { génome }\end{array}$ \\
\hline Variables entre environnements & Interaction gène $\times$ environnement \\
\hline
\end{tabular}

\section{Le gène de nanisme lié au sexe ( $\mathrm{dw}$ )}

Le gène de nanisme lié au sexe de la poule $(d w)$ a été décrit par Hutt (1949). Il est apparu par mutation dans diverses populations (Guillaume 1976). On a montré que le gène $d w$ abaisse le taux plasmatique de tri-iodothyronine (T3) et d'IGF 1, et augmente légèrement celui de la thyroxine (T4) et de l'hormone de croissance $(\mathrm{GH})$. La déiodination dans le foie est réduite malgré la présence de déiodinase dans les cellules hépatiques, et l'attachement de l'hormone de croissance aux cellules hépatiques est réduit chez les oiseaux nains. Ces modifications aident à interpréter l'effet dépressif du gène de nanisme sur la croissance du fait de l'influence de T3, GH et IGF 1 sur celle-ci. Les conséquences sur la production d'œufs sont plus difficiles à expliquer.
Figure 1. Effets du gène dw (écarts en \% au génotype $D w+$ ) chez les poules de type "ponte» ou " chair".

(): nombres de rapports tirés de la littérature

(1): g. d'œuts parg. d'aliment consommé pour les pondeuses, par douzaine d'ouf pour les reproductrices chair.

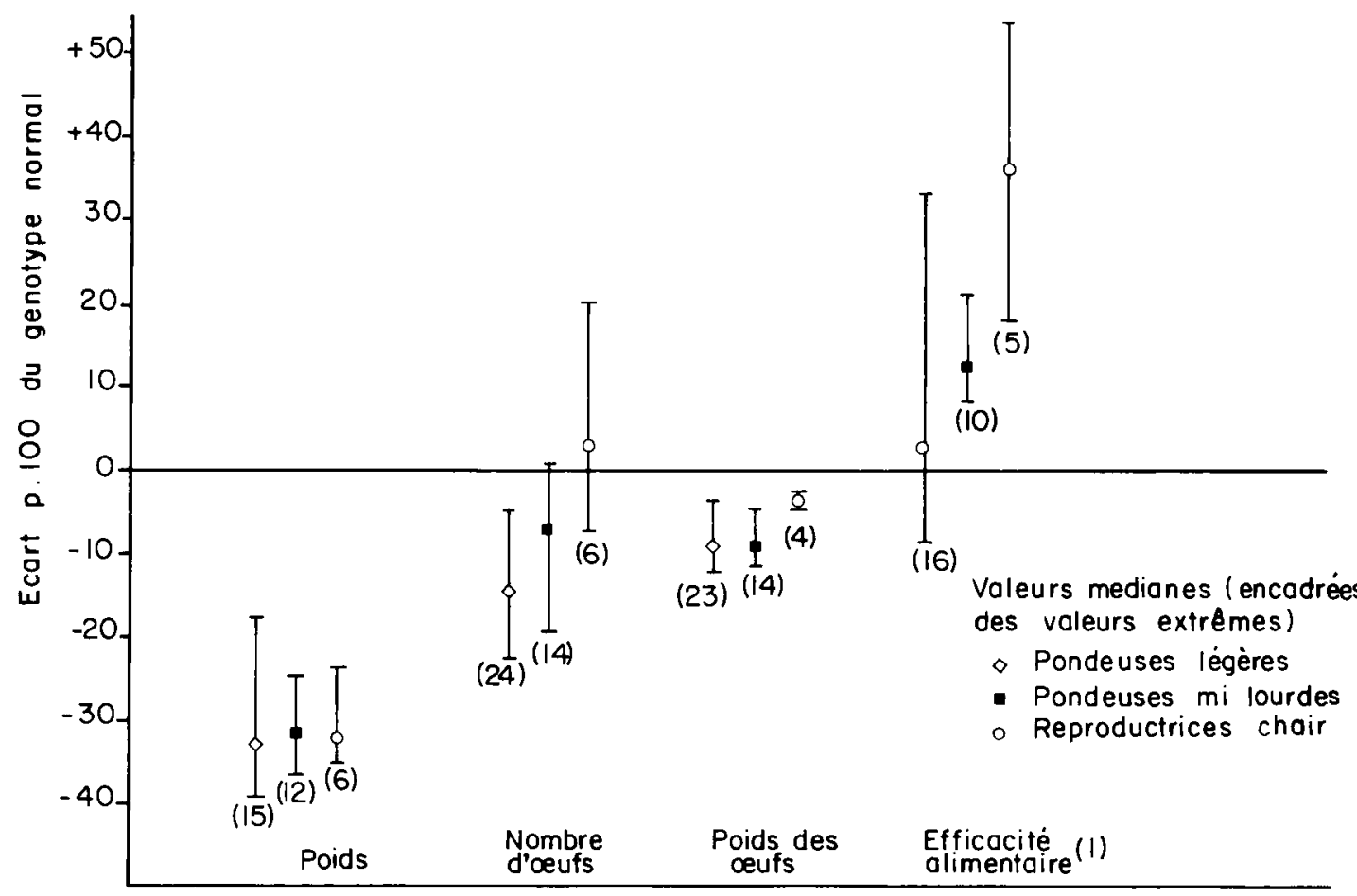


que le poids corporel est moins diminué par le gène de nanisme dans une lignée sélectionnée pour un poids élevé que dans une lignée sélectionnée en sens contraire. Cependant ceci n'apparaît pas dans toutes les comparaisons entre populations lourdes et légères (figure 1).

\section{b / Caractères de production d'aufs}

L'effet sur certains critères de production d'œufs diffère chez les lignées «pondeuses» (Leghorn ou type mi-lourd à cufs bruns) et chez les lignées " chair " (figure 1).

L'âge moyen au premier œuf est retardé de quelques jours à deux semaines. Le poids de l'œuf est réduit corrélativement à la diminution du poids corporel, mais moins en moyenne dans les lignées " chair » que dans les lignées " ponte». Le nombre d'œufs est abaissé dans les populations "pondeuses", et davantage dans le type Leghorn plus léger que dans les types à œuf brun, mais il ne subit pas de réduction dans les lignées « chair ». En conséquence, l'efficacité alimentaire des reproductrices chair est améliorée de l'ordre de $25 \%$ (par gramme d'œuf) par le gène de nanisme, du fait que la masse d'œufs n'est affectée que par la diminution modérée du poids moyen de l'œuf. Chez les types «ponte»mi-lourds, malgré une réduction du nombre d'œufs, l'efficacité alimentaire des poules $d w$ comparées aux poules de taille normale fait apparaître un gain moyen d'environ $13 \%$; chez les Leghorns le gain moyen est faible, mais l'efficacité est cependant plus souvent améliorée que détériorée.

Les œufs anormaux (à deux jaunes ou sans coquille) sont presque supprimés par le gène $d w$ (Guillaume 1976, Mérat 1984). L'absence d'effet défavorable de ce gène sur l'intensité de ponte dans les souches lourdes en est vraisemblablement une conséquence. Jaap et Clancy (1968) ont montré que les poules de type " chair » ont un nombre excessif de follicules ovariens de grande taille, entraînant un pourcentage élevé d'œufs anormaux et un taux de ponte réduit, alors que les poules de type "ponte» ont un nombre moindre de ces follicules, plus compatible avec une ponte normale. Dans les deux cas, le gène $d w$ lié au sexe réduit le nombre des grands follicules ; dans les populations "chair», il le ramène à des valeurs proches de l'optimum, entraînant la suppression des œufs anormaux, alors que dans les populations de pondeuses il abaisse ce nombre au-dessous du niveau permettant la production d'œufs maximum.

Un autre effet de $d w$ est de raccourcir la longueur des séries de ponte, ceci étant associé à un allongement de l'intervalle de temps entre ovipositions successives par comparaison avec le gène $D_{W^{+}}$.

De nombreux auteurs (voir la revue de Mérat, 1984) ont montré que le gène $d w$ réduit - souvent de plus de moitié - le.pourcentage d'œufs cassés en batterie, aussi bien dans les souches légères que dans les souches lourdes. Il n'a pas d'effet appréciable sur l'épaisseur des coquilles ou le poids spécifique des œufs qui lui est lié. La réduction de fréquence des œufs cassés peut être attribuée au poids corporel plus faible de la poule, car tout poids appliqué sur le plancher des cages augmente le choc mécanique subi par l'œuf pondu. En outre, la hauteur de chute de l'œuf à l'oviposition peut être moindre du fait des tarses plus courts des poules naines. Le tempérament réputé plus calme des poules $d w$ pourrait éventuellement aussi jouer un rôle.

Il n'a pas été trouvé d'effet du gène $d w$ sur la consistance de l'albumen de l'œuf (unités Haugh); nous avons montré d'autre part que, dans les triglycérides du jaune, le pourcentage d'acide linoléique passe d'une valeur moyenne de $14,8 \%$ chez les femelles $D_{W^{+}}+$à $22,9 \%$ chez les poules $d w$ tandis que les valeurs correspondantes pour l'acide oléique sont respectivement de 51,8 et 43,3 .

\section{c / Mortalité}

Pour la mortalité globale, la majorité des résultats ne font pas apparaître de différences significatives entre les poules $D w^{+}$et $d w$; l'avantage semble être plus souvent en faveur de ces dernières que l'inverse. Quisenberry (1972, cité par Guillaume 1976) comparant des poules naines et normales auxquelles on distribue des aliments à quatre taux protéiques différents (de 22 à $14 \%$ ) observe pour les pondeuses naines une survie meilleure au taux protéique le plus élevé et moins bonne au plus faible taux.

Concernant des maladies spécifiques, le tableau 2 résume les effets trouvés associés au gène $\mathrm{dw}$ par comparaison au génotype normal.

\section{d / Fertilité et taux d'éclosion}

La fertilité n'a pas été trouvée significativement différente pour des reproductrices $D_{W^{+}}$ et $d w$ accouplées à des mâles semblables, à la fois dans des lignées "chair» ou «ponte» (revue de Guillaume 1976, Mérat 1984)

Dans les souches lourdes, les reproductrices nanifiées présentent un léger avantage pour le taux d'éclosion (Ricard 1976, revues par Guillaume 1976 et Mérat 1984), attribuable à la faible fréquence des œufs anormaux.

\section{2 / Réponses des animaux nains à des facteurs de l'environnement}

Les cas, rapportés dans la littérature, d'une réponse différente à des facteurs du milieu associée au gène $d w$, sont en nombre limité. Pour certains d'entre eux, une base physiologique peut être proposée. Un catabolisme protéique plus rapide et un accroissement de la gluconéogénèse ont été suggérés par Guillaume (1976). En outre, la masse d'œufs exportée par les poules naines est plus importante si on la rapporte au poids corporel.

En fait, d'après certains résultats de la littérature les poules $d w$ répondaient davantage à une augmentation des protéines totales ou de la méthionine que des poules $D w^{+}$; d'autres résultats ne montraient pas d'interaction gène $\mathrm{x}$ ration.

Le niveau énergétique de l'aliment n'affecte pas la pondeuse naine de façon spécifique. Pour le métabolisme lipidique, il est modifié par le gène de nanisme, avec une lipolyse réduite (Guillaume 1976) et une moindre syn- 
Tableau 2. Effet de dw sur la réponse à des maladies spécifiques et sur la réponse immunitaire.

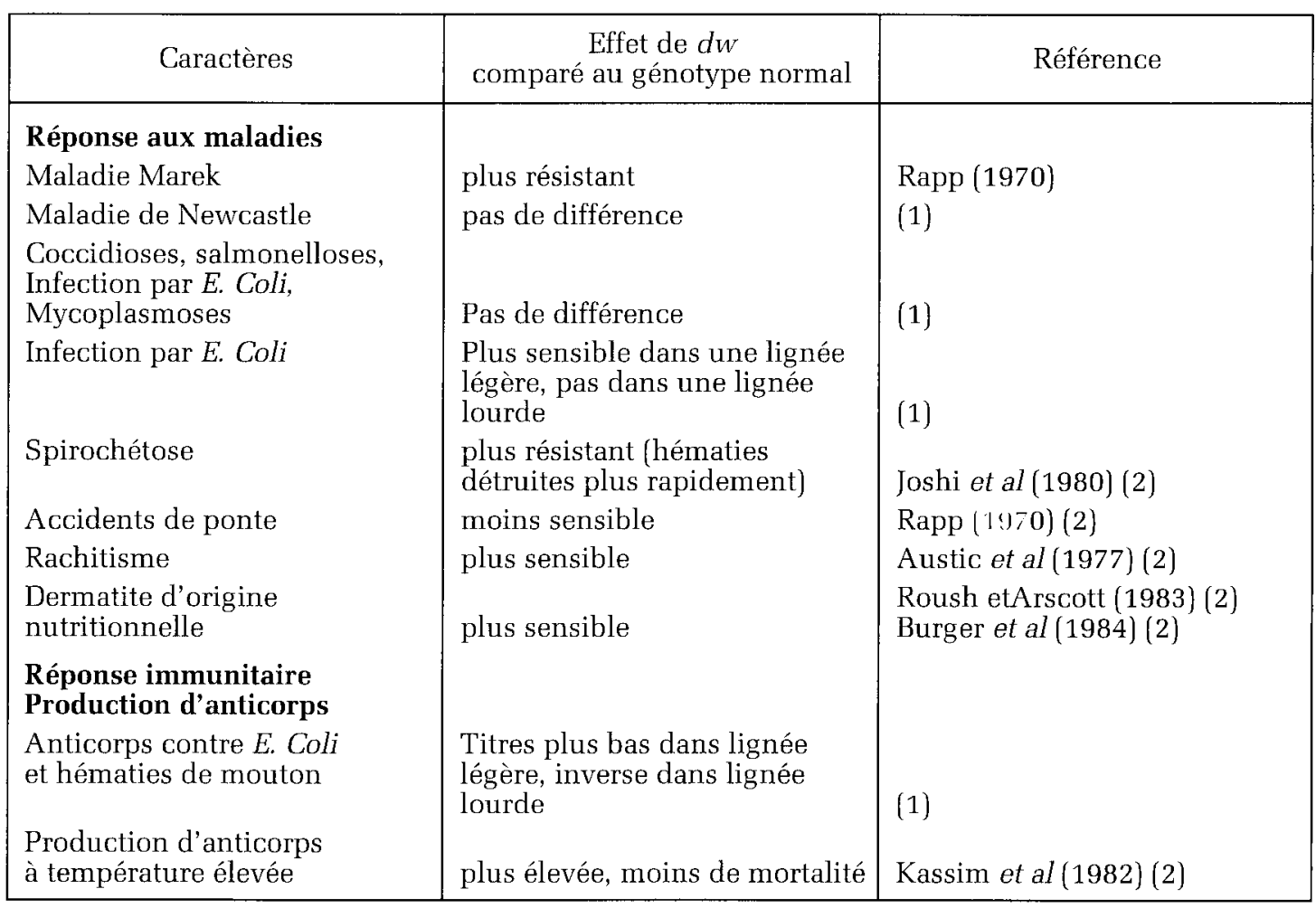

(1) Références citées dans Mérat (1984)

(2) Références citées dans Mérat (1990)

thèse des acides gras libres est vraisemblable. L'effet d'une augmentation du taux de lipides de la ration sur le rythme de ponte des poules naines est en tout cas faible.

Concernant le métabolisme du calcium chez les pondeuses, une proportion plus élevée de cet élément paraît être tirée de l'aliment par la pondeuse naine durant la formation de la coquille (Bordas et Mérat 1976). Les pondeuses naines légères ont été trouvées affectées par un faible niveau de calcium alimentaire, et les mini-pondeuses bénéficiaient peut-être plus que les autres d'une alimentation calcique séparée.

Relativement à l'éclairement, il n'y a pas de raison de s'attendre a priori à une réponse particulière des poules $d w$. Cependant, elles ont un plus long intervalle de temps moyen entre ovipositions successives; de ce fait, elles peuvent être relativement avantagées par des nycthémères supérieurs à 24 heures. Ceux-ci évitent les interruptions des séries de ponte, provenant du décalage entre le rythme interne de la poule et le cycle de 24 heures. Une réponse dans ce sens - d'amplitude toutefois limitée a effectivement été observée sur des pondeuses $d w$ mi-lourdes en nycthémères de 26 et 27 heures.

Il est connu qu'aux températures ambiantes élevées un faible poids corporel augmente la thermolyse par suite de l'augmentation du rapport de la surface au poids corporel. Cependant, dans le cas du gène $d w$ les choses ne sont peut être pas si simples; Horst et Petersen (1979) suggèrent l'existence d'un optimum différent du poids de la poule pour les génotypes
$D w^{+}$et $d w$ selon la température ambiante. A des températures relativement basses, plusieurs auteurs ont observé une diminution de la consommation alimentaire des poules naines, contrairement aux poules $D W^{+}$; par contre, nos propres données non publiées n'indiquent pas de réduction de ponte pour les poules $d w$ à $18^{\circ} \mathrm{C}$ comparativement à $25^{\circ} \mathrm{C}$. Aux températures élevées, divers résultats montrent un nombre et un poids moyen des œufs légèrement moins diminués pour les poules naines que pour leurs homologues normales par référence aux températures modérées, mais certains autres résultats ne font pas apparaître d'avantage relatif des pondeuses $d w$ en ambiance chaude.

Enfin, on n'a pas mis en évidence d'interaction significative entre génotype au locus $D w$ et mode d'élevage (sol ou cages) ou densité d'occupation des cages.

\section{3 / Interaction avec le reste du génome et utilisation du gène $d w$ pour la production d'œufs}

Dans les souches de pondeuses, l'intérêt potentiel du gène de nanisme réside dans la réunion d'un meilleur taux de conversion alimentaire pour le production d'œufs, d'une économie de 25 à $30 \%$ sur la consommation d'aliment jusqu'à la maturité sexuelle, d'une réduction de la fréquence des ceufs cassés et mous ou sans coquille, d'un volume de déjections de $20 \%$ plus faible par poule, et d'un moindre besoin en surface par pondeuse. Les programmes de rationnement sont évités dans les 
souches mi-lourdes de type œuf brun. La proportion plus élevée d'acides grảs polyinsaturés dans le jaune d'œuf peut être légèrement favorable pour l'alimentation humaine. Sont opposés à ces avantages un abaissement du poids moyen de l'œuf, et en général du nombre d'œufs dans les lignées légères. Il est donc nécessaire de rechercher les conditions de nature à optimiser le bilan d'ensemble du gène.

\section{a / Poids corporel optimal}

La figure 1 montre que le gène de nanisme est plus avantageux en moyenne dans des populations de type " cuf brun » que dans des lignées Leghorn plus légères. A l'intérieur de chaque type, la figure 2 montre, à partir de données bibliographiques, le poids adulte moyen d'une population d'une part, et d'autre part, l'écart, en pourcentage du génotype normal, apporté par le gène $d w$ respectivement pour le nombre d'œufs, leur poids moyen et le taux de conversion alimentaire.

En dépit de l'hétérogénéité des données, comprenant en particulier plusieurs sources distinctes de mutation, celles-ci ne s'écartent pas significativement d'une régression linéaire unique; plus le poids corporel moyen est faible, plus l'effet dépresseur du gène $d w$ sur le nombre des œufs et leur poids moyen est important, et moins l'effet sur l'indice de consommation est favorable. En moyenne ce dernier effet commence à être avantageux lorsque le poids des poules $d w$ adultes excède $1 \mathrm{~kg}$. Ceci semble en accord avec l'observation d'une corrélation phénotypique positive à l'intérieur de lignées nanifiées de type ponte entre le nombre d'œufs et le poids corporel (voir Mérat 1984). Ces résultats rejoignent la suggestion selon laquelle un format suffisant est requis pour les mini-pondeuses.

\section{b / Amélioration génétique de la production d'œufs}

La figure 1 montre qu'il existe des populations, y compris en race Leghorn, où l'effet du gène $d w$ est plus favorable que la moyenne, et cet avantage peut être augmenté par une sélection à long terme pour la ponte. Les résultats de Yoo et al (1984) suggèrent de combiner l'emploi du gène de nanisme avec une sélection pour un court intervalle entre ovipositions. Leenstra et al (1986) ont proposé d'introduire le gène $d w$ dans des lignées produisant un pourcentage élevé d'ovulations multiples (de façon comparable aux lignées «chair»): dans ce contexte, le gène $d w$ rapprocherait le taux d'ovulation de la valeur optimale. Le succès d'une telle formule dépendrait cependant de l'absence de réponses liées défavorables à la sélection pour des ovulations multiples.

Les inconvénients associés au gène $d w$ pourraient être minimisés par l'hétérosis ; c'est du moins le cas d'un croisement Leghorn x Australorp (Yoo et al 1980, cités par Horst 1981). Si ceci avait un caractère général, les perspectives de l'utilisation du gène de nanisme pour la production d'œufs pourraient être plus optimistes que ce que font prévoir les résultats obtenus en lignées pures.
Figure 2a. Effet du gène dw sur le nombre d'œufs dans des lignées de type "ponte" selon le poids corporel des poules dw (à partir de 19 résultats de la littérature).

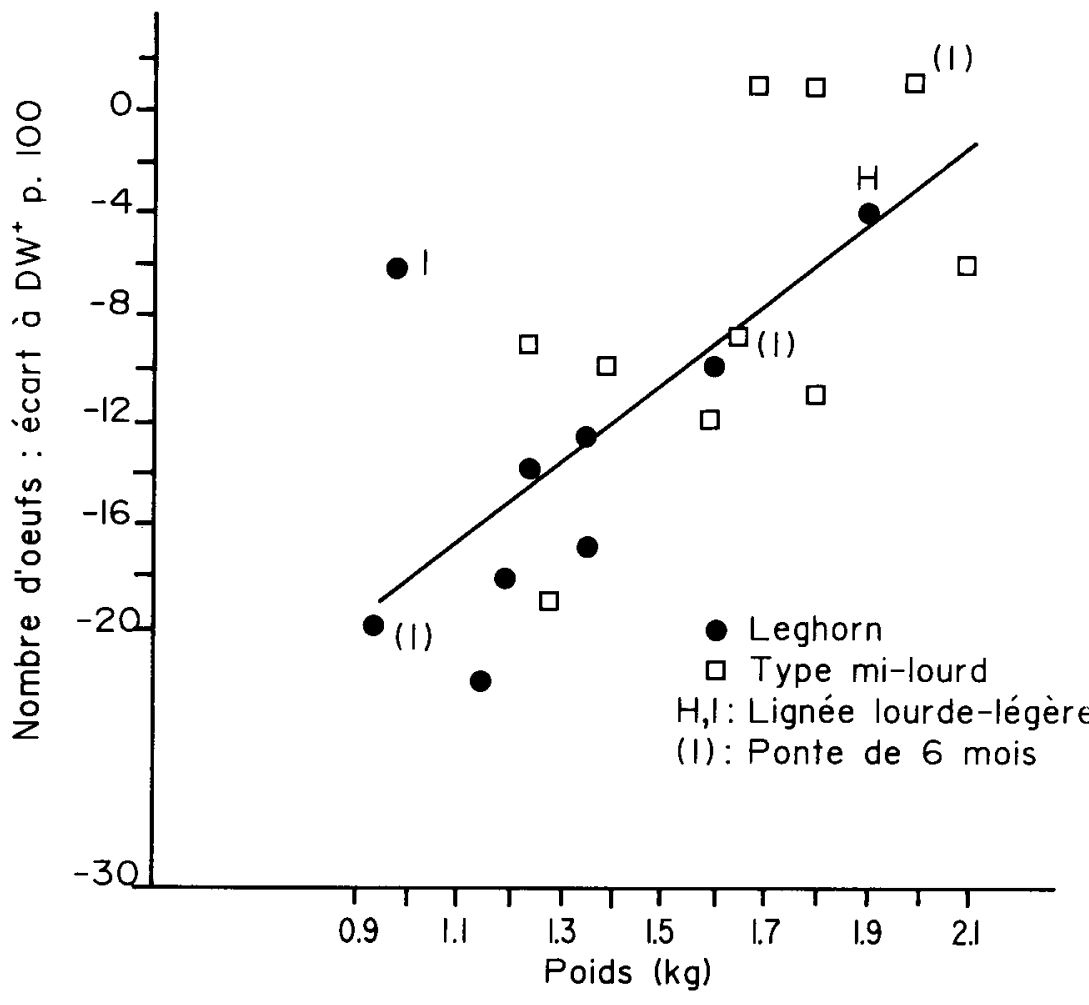

Figure 2b. Effet du gène dw sur le poids moyen des œufs selon le poids corporel des poules dw (à partir de 22 résultats de la littérature).

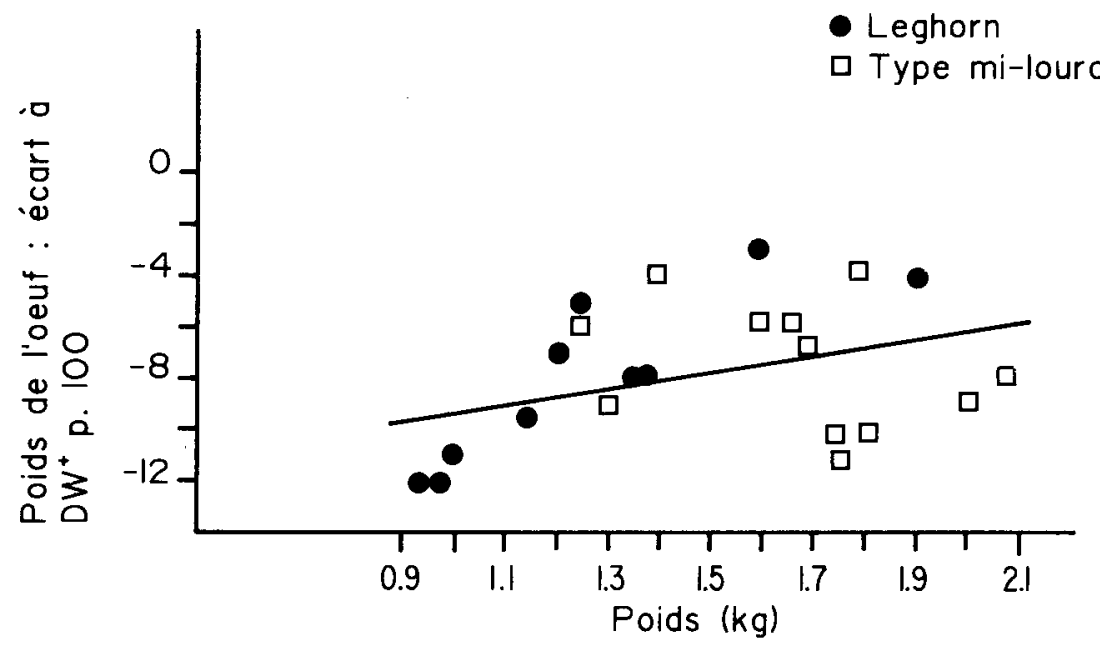

\section{c / Eviter une sous-consommation alimentaire des pondeuses naines}

S'ajoutant à une utilisation médiocre des protéines par les mini-pondeuses, il est à prévoir que leur ingestion de protéines est plus faible relativement à la masse d'œufs produite que pour des poules de plus grande taille. De plus, une tendance à la sous-consommation est suggérée pour les oiseaux nains. Les poussins nains mettent plus longtemps que les autres pour ingérer une moindre quantité d'aliment. La consommation alimentaire des nains décroît 
Figure 2c: Effet du gène dw sur l'efficacité alimentaire pour la production d'œuts selon le poids corporel des poules dw (à partir de 19 résultats de la littérature).

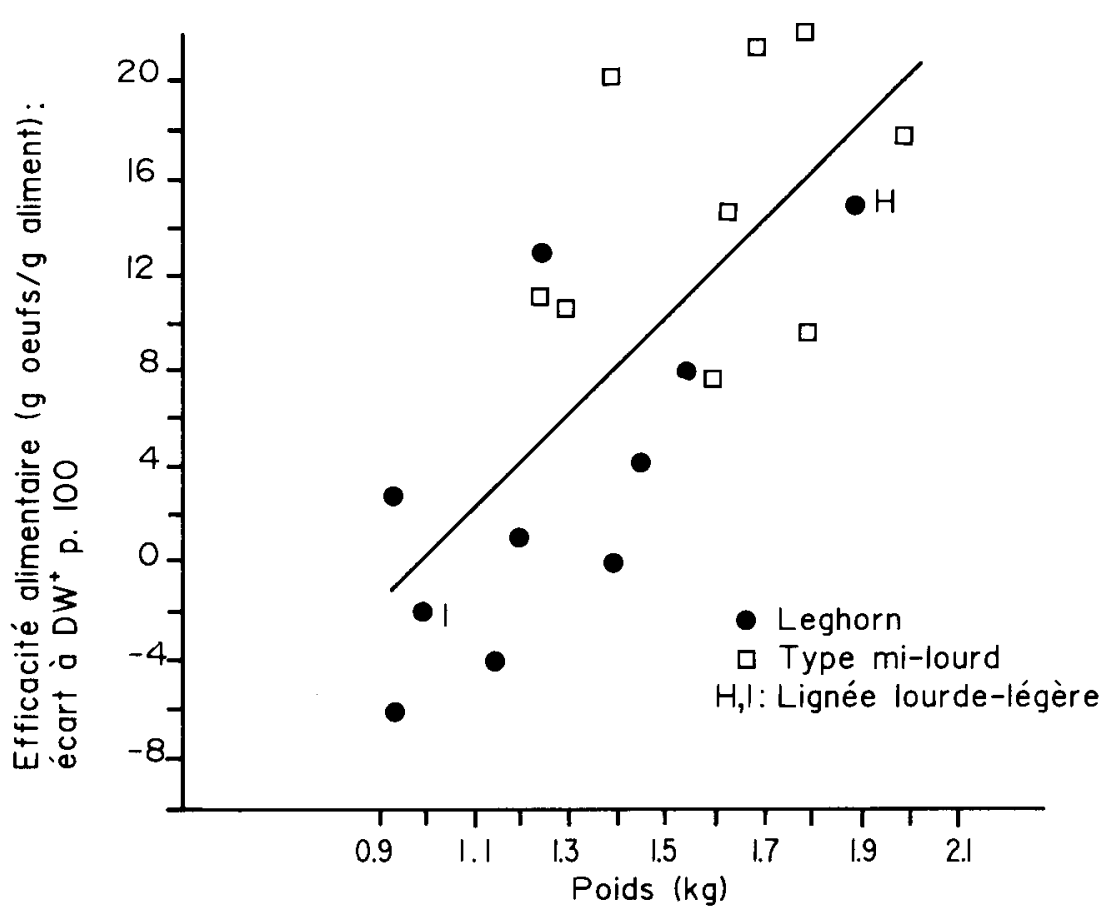

Le poids à l'abattage des poulets issus d'un croisement femelle nanifiée $\times$ mâle standard est très légèrement inférieur à celui des poulets standards, l'adiposité étant identique. au lieu d'augmenter aux basses températures. Le gène "Cou nu » augmente moins l'ingestion de nourriture chez les poules naines que chez celles de taille normale; il a cependant un effet bénéfique sur le taux de ponte des premières (Mérat, données non publiées). En outre, il a été montré qu'une consommation insuffisante d'aliment et en particulier de protéines affecte la longueur des séries de ponte d'une façon analogue à l'effet du gène $d w$ dans les populations de type ponte. L'opinion selon laquelle une augmentation de l'ingestion alimentaire des pondeuses naines peut améliorer leur production a été avancée par divers auteurs.

\section{d / Situations particulières}

Des situations particulières peuvent maximiser les avantages ou minimiser les inconvénients associés au gène $d w$, par exemple lorsque le poids des œufs a une importance secondaire ou nulle (fabrication d'ovoproduits), si l'on utilise une lignée maternelle nanifiée dans un croisement "ponte ", ou lorsque des économies sur le coût des aliments sont d'une importance particulière, ce qui est le cas des pays en développement.

L'emploi du gène de nanisme lié au sexe pour la production d'œufs devrait être mis en balance avec le résultat de l'abaissement du poids corporel par une sélection «classique» (French et Nordskog 1973, cités par Guillaume 1976). Une bonne partie des effets associés au gène $d w$ peuvent être obtenus grâce à une réduction de taille non liée à ce gène. Cependant, on ne peut exclure la possibilité de réponses liées défavorables dans une sélection à long terme pour un faible poids corporel.

\section{4 / Utilisation du gène $d w$ en production " chair "}

La réduction du poids adulte par le gène de nanisme lié au sexe et sa récessivité permettent l'obtention de poulets de chair de taille normale à partir de reproductrices nanifiées croisées à des coqs $D w^{+} D w^{+}$. Les besoins alimentaires de la reproductrice naine sont abaissés à la fois pendant sa période d'élevage et celle de la reproduction (environ $25 \%$ par g d'oeuf, davantage par douzaine d'oeufs). Les effets sur la ponte, le taux d'éclosion et la viabilité dans les souches lourdes sont plutôt favorables, et on peut prévoir un nombre de poussins par mère accru de $4 \%$ en moyenne, principalement du fait de la quasi suppression des oeufs à deux jaunes, mous ou cassés. Ce dernier point serait particulièrement important dans le cas d'une reproduction en batterie. La diminution de la surface nécessaire par poule peut aller jusqu'à $40 \%$. La fertilité des mini-poules avec des coqs lourds en accouplement normal n'est pas considérée en général comme une difficulté majeure, quoiqu'elle puisse être diminuée si la disproportion de taille est trop grande. La thermotolérance meilleure notée pour des poules naines peut concerner particulièrement des populations lourdes de type «chair». Ces avantages doivent être comparés à l'effet légèrement négatif sur la croissance des poulets d'un croisement terminal. La mère naine produit des oeufs de 3 à $4 \mathrm{~g}$ moins lourds en moyenne, d'où un petit effet dépresseur sur le début de la croissance des descendants. En récapitulant 14 résultats de la littérature, Mérat (1984) a trouvé une valeur médiane pour la diminution du poids à l'abattage de la descendance femelle d'environ $0,7 \%$ ou 10 à $15 \mathrm{~g}$. L'allèle $d w$ est d'autre part incomplètement récessif, de sorte que le poids à l'abattage des mâles $D_{w^{+}} d w$ n'est pas tout à fait égal à celui des homozygotes normaux. En récapitulant les mêmes 14 rapports, la déviation médiane correspondante par rapport à l'homozygote normal était proche de $2,8 \%$, représentant 40 à $60 \mathrm{~g}$ selon l'âge à l'abattage. D'après la même revue, il n'y avait pas d'effet décelable associé au génotype $D w+d w$ sur l'efficacité alimentaire ni sur l'adiposité. Des situations particulières peuvent maximiser les avantages: production de poulets abattus tôt, où les économies sur le coût du poussin d'un jour ont une influence relative plus grande sur le prix de revient total ; production « chair » dans des pays en développement, où les économies sur le coût de l'aliment soient particulièrement importantes.

\section{Autres gènes réduisant la taille}

Deux autres gènes réduisant la taille corporelle chez les volailles méritent d'être cités.

\section{1 / Nanisme autosomal (adw)}

Ce gène récessif, trouvé en race Leghorn, réduit le poids du corps d'environ $30 \%$, abaisse le taux d'éclosion, retarde la maturité sexuelle, entraîne une réduction de $10 \%$ du nombre d'oeufs, mais s'accompagne d'une bonne viabilité (Cole 1973). Son utilisation 
dans les troupeaux de pondeuses pour diminuer les besoins alimentaires nécessiterait d'introduire l'allèle dans toutes les lignées parentales d'un croisement. Leenstra et Pit (1984) proposent de l'utiliser pour réduire la taille d'une lignée de mâles « chair » accouplés à des femelles $d w$ (nanisme lié au sexe) pour éviter des difficultés de fécondation. Dans une population de type " chair » étudiée par ces auteurs, le génotype adwadw était associé à des anomalies des pattes affectant la viabilité, mais leurs données suggéraient une récessivité complète du gène $a d w$ pour la croissance. L'emploi éventuel de ce gène dépend de recherches ultérieures pour savoir si sa relation avec les anomalies des tarses est permanente ou causée par un linkage ou par une interaction entre des gènes particuliers.

\section{2 / Nanisme type « Bantam » $\left(d w^{B}\right)$}

Plusieurs auteurs ont montré l'existence d'un gène récessif lié au sexe pour une taille réduite chez des races de type Bantam. Ce gène a été localisé à proximité des loci $\mathrm{S}$ et $\mathrm{K}$. Custodio et Jaap (1973) ont suggéré que ce facteur était un allèle $\left(d w^{B}\right)$ au locus $D w$. D'après Shivaprasad et Jaap (1977), $d w^{B}$ s'accompagne seulement d'une réduction de $10 \%$ du poids et d'une ponte supérieure à celle des poules $d w$. Coquerelle et Mérat (1979), comparant des oiseaux porteurs d'un facteur lié au sexe similaire issu de la race Ardennaise naine à des animaux de taille normale de même origine, l'ont trouvé associé à une réduction du poids corporel adulte de $13 \%$, du poids moyen de l'oeuf de $4 \%$, pas de diminution du nombre d'oeufs et un gain de $6 \%$ sur le taux de conversion alimentaire pour la production d'oeufs. Apparemment en accord avec ces résultats, Yoshida et Saito (1983), pour un facteur analogue lié au locus $\mathrm{K}$, ont observé une diminution du poids adulte de 8 à $17 \%$, un poids moyen d'oeufs abaissé de 3-4\%, sans modification du nombre d'oeufs.

Un tel gène lié au sexe a une valeur limitée pour améliorer l'efficacité alimentaire pour la ponte par comparaison avec la sélection pour réduire le poids corporel. Cependant, son introduction dans la lignée mâle d'un croisement pourrait être relativement simple.

Cet article est adapté, pour l'essentiel, de celui de Mérat P., 1990. Pleiotropic and associated effects of major genes. In Crawford R.D. (Ed), Poultry Breeding and genetics, 429-467, Elsevier, Amsterdam.

\title{
Principales références bibliographiques
}

\author{
(La liste complète est disponible auprès de l'auteur)
}

COLE R.K., 1973. An autosomal dwarfism in the fowl. Poultry Sci. 52 : 2012-2013 (Abstract).

COQUERELLE G., MERAT P., 1979. Gène de réduction de taile lié au sexe chez la poule (dwB) : effet sur la ponte et la consommation d'aliment. Ann. Génét. Sél. Anim. 11: $267-269$

CUSTODIO R.W.S., JAAP R.G., 1973. Sex-linked reduction of bdy size in Golden Sebright Bantams. Poultry Sci. $52: 204-210$.

GUILLAUME J., 1976. The dwarfing gene dw, its effects on anatomy, physiology, nutrition, management. Its application in poultry industry; World's Poultry Sci. J. $32: 285-303$.

HORST P., 1981. Breeding perspectives for fowl with improved adaptability to the tropics. 4* Congres International SABRAO, 11-27

HORST P., PETERSEN J., 1978. Importance of the dwarf gen (dw) for the breeding of laying hens. $5^{\circ}$ Conf. Avic. Europ. (Malte) $1: 284-303$.

HORST P., PETERSEN J., 1979. Der Effekt des dwarfgenes auf das Akklimatizationsvermögen von Legehennen an hole Umwelt-temperaturen. Archiv. Geflügel. 43: 242.

HUTT F.B., 1949. Genetics of the fowl McGraw-Hill Book Co., New-York.

JAAP R.G., CLANCY J.A., 1968. Reproductive idiosyncrasies of the broiler pullet. 3" Conf. Avic. Europ., Jérusalem ], 74-79.

LEENSTRA F.R., PITT R., 1984. The autosomal dwarf as broiler sire mated to normal and sex-linked dwarf broiler dams: performance of progeny. $17^{\circ}$ Congrès Mondial d'Aviculture (Helsinki), 140-142.

LEENSTRA F.R., Van MIDDELKOOP J.H., VERELIJKEN P.G., ABPLANALP H., 1986. Influence of the sex-linked dwarfing gene on yolk and egg production in normal and superovulating laying hens. Archiv Geflügel. 50 : 65-68.

MERAT P., 1984. The sex-linked dward gene in the broiler chicken industry. World's Poultry Sci. J., 40 : 1018.

MERAT P., 1990. Pleiotropic and associated effects of major genes. In Crawford R.D. Ed., Poultry Breeding and Genetics, 429-467, Elsevier, Amsterdam

RICARD F.H., 1976. Bases scientifiques de l'utilisation du gène de nanisme dw dans la production du poulet de chair. $5^{\text {e }}$ Conf. Avic. Europ. (Malte) $1: 263-283$.

SCANES G.G., HARVEY S., MARSH J.A., KING D.B 1984. Hormones and growth in poultry. Poultry Sci. 63 : 2062-2074

SHIVA-PRASAD H.L., JAAP R.G., 1977. Egg and yolk production as influenced by liver weight, liver lipid and plasma lipid in three strains of small bodied chickens. Poultry Sci., 56 : 1384-1390.

YOO B.H., SHELDON B.L., PODGER R.N., 1984. Increase in oviposition interval due to the sex-libked dwarf gene in White Leghorns ans Australorps. Brit. Poultry Sci. 25 : 119-126.

YOSHIDA S., SAITO K., 1973. The effects of sex-linked bantam gene dwB on economic traits in Sebright Bantam crosses. $5^{*}$ Congrès Mondial de Production Animale, 2 : $115-116$. 


\section{Summary}

Associated effects and utilization of major genes reducing body size in the fowl.

Among the major genes reducing body size in the domestic fowl, the sex-linked dwarfing gene $\mathrm{dw}$ was the subject of numerous studies and of applications on an industrial scale.Its recessiveness ant the fact that it lowers the body weight of the bird by one third and its feed consumption by $20-25 \%$ led to its utilization in female broiler parental lines, giving normal-sized broilers when crossed with a standard broiler male line. For egg production, the use in the industry is more difficult, due to the fact that in egg production stocks, especially those of smaller body size (leghorns), the introduction of this gene causes a lowering of egg number and mean egg weight.
In spite of this, in medium-size brown egg type lines the dw gene improves feed efficiency, avoids feed restriction programs and reduces the frequency of cracked and of softshelled eggs. Hence research work has been done so as to better estimate the optimal conditions for the use of «mini-layers » : preferable incorporation of the gene into mediumsized laying stocks, avoidance of feed underconsumption, utilization in production conditions where feed savings are relatively most important.

Another sex-linked mendelian factor from Bantam breeds, lowering body weight by only 10-15\% without any deleterious effect on egg numbers, could be useful in specific crosses.

MERAT P., 1990. Effets associés et utilisation de gènes majeurs réduisant la taille chez la poule domestique. INRA Prod. Anim., 3 (2), 151-158 